\title{
Molecular alterations in pediatric brainstem gliomas
}

\author{
Porkholm, Mikaela
}

2018-01

Porkholm , M , Raunio, A, Vainionpää , R, Salonen , T, Hernesniemi , J , Valanne , L , Satopää , J , Karppinen , A , Oinas , M , Tynninen , O \& Kivivuori , S-M 2018 , ' Molecular alterations in pediatric brainstem gliomas ' , Pediatric Blood \& Cancer , vol. 65 , no. 1 , 26751 . https://doi.org/10.1002/pbc.26751

http://hdl.handle.net/10138/302389

https://doi.org/10.1002/pbc.26751

publishedVersion

Downloaded from Helda, University of Helsinki institutional repository.

This is an electronic reprint of the original article.

This reprint may differ from the original in pagination and typographic detail.

Please cite the original version. 


\title{
Molecular alterations in pediatric brainstem gliomas
}

\author{
Mikaela Porkholm ${ }^{1}$ (D) | Anna Raunio ${ }^{2}$ | Reetta Vainionpää3 ${ }^{3}$ Tarja Salonen $^{3}$ | \\ Juha Hernesniemi $^{4}$ | Leena Valanne ${ }^{5}$ | Jarno Satopää4 ${ }^{4}$ Atte Karppinen ${ }^{4}$ \\ Minna Oinas $^{4}$ | Olli Tynninen ${ }^{2}$ | Virve Pentikäinen ${ }^{6}$ | Sanna-Maria Kivivuori ${ }^{1}$
}

${ }^{1}$ Department of Children and Adolescents, Helsinki University Hospital and University of Helsinki, Helsinki, Finland

2Department of Pathology, University of Helsinki and HUSLAB, Helsinki, Finland

${ }^{3}$ Laboratory of Pathology and Genetics, HUSLAB, Helsinki, Finland

${ }^{4}$ Department of Neurosurgery, Helsinki University Hospital and University of Helsinki, Helsinki, Finland

${ }^{5}$ Department of Radiology, Helsinki University Hospital and University of Helsinki, Helsinki, Finland

${ }^{6}$ Division of Hematology-Oncology and Stem Cell Transplantation, Children's Hospital, Helsinki University Hospital, Helsinki, Finland Correspondence

Mikaela Porkholm, c/o Sanna-Maria Kivivuori, Children's Castle, Helsinki University Central Hospital, P.O. Box 280, FIN-00029 HUS Finland. Email:mikaela.porkholm@helsinki.fi

\begin{abstract}
Background: Diffuse intrinsic pontine gliomas (DIPGs) have a dismal prognosis. Previously, diagnosis was based on a typical clinical presentation and magnetic resonance imaging findings. After the start of the era of biopsies, DIPGs bearing H3 K27 mutations have been reclassified into a novel entity, diffuse midline glioma, based on the presence of this molecular alteration. However, it is not well established how clinically diagnosed DIPG overlap with H3 K27-mutated diffuse midline gliomas, and whether rare long-term survivors also belong to this group.

Methods: We studied tumor samples obtained at diagnosis or upon autopsy from 23 children, including two long-term survivors. Based on clinical, radiological, and histological findings, all tumors were previously diagnosed as DIPGs. All samples were analyzed for genetic alterations by next-generation sequencing (NGS) and for protein expression by immunohistochemistry (IHC).

Results: H3 K27 was mutated in NGS or IHC in 20 patients, excluding both long-term survivors. One of these long-term survivors harbored a mutation in IDH1, formerly considered to be an alteration absent in pediatric diffuse brainstem gliomas. Other altered genes in NGS included TP53 (10 patients), MET and PDGFRA (3 patients each), VEGFR and SMARCA4 (2 patients each), and PPAR $\gamma$, PTEN and EGFR in 1 patient, respectively. IHC revealed cMYC expression in 15 of 24 (63\%) of all samples, exclusively in the biopsies.

Conclusions: Eighty-seven percent of the tumors formerly diagnosed as DIPGs could be reclassified as H3 K27-mutated diffuse midline gliomas. Both long-term survivors lacked this alteration. Contrary to former conceptions, IDH1 mutations may occur also in pediatric brainstem gliomas.
\end{abstract}

KEYWORDS

Brainstem glioma, diffuse intrinsic pontine glioma, next-generation sequencing, pediatric

\section{1 | INTRODUCTION}

Diffuse intrinsic pontine gliomas (DIPGs) are rare and highly malignant tumors that typically arise in children and adolescents. They have a notorious prognosis, with most patients deceasing within a year from diagnosis ${ }^{1}$ and less than $10 \%$ surviving beyond 24 months. ${ }^{2}$ Surgery is precluded, irradiation alleviates the symptoms only transiently, and chemotherapies lack any significant effect on the outcome. ${ }^{1}$ DIPG diagnosis has traditionally been made on clinical grounds based on characteristic symptoms and magnetic resonance imaging (MRI) findings.

Abbreviations: DIPG, diffuse intrinsic pontine glioma; IHC, immunohistochemistry; MRI, magnetic resonance imaging; MVD, microvessel density; NGS, next-generation sequencing; OS, overall survival; $\mathrm{PCR}$, polymerase chain reaction; WHO, World Health Organization
Recent molecular studies have revealed that most malignant gliomas arising in the midline structures of the central nervous system-brainstem, thalamus, and spinal cord-harbor a highly specific point mutation, $\mathrm{K} 27 \mathrm{M}$, in histone $3.3,3.2$, or $3.1 .^{3-7}$ The dismal prognosis specifically concerns those tumors bearing $\mathrm{H} 3 \mathrm{~K} 27$ mutations. ${ }^{2}$ In the revised 2016 edition of the World Health Organization (WHO) brain tumor classification, these neoplasms constitute a new entity termed as diffuse midline glioma, $\mathrm{H} 3 \mathrm{~K} 27 \mathrm{M}$-mutant. ${ }^{8} \mathrm{H} 3$ K27M-mutated DIPGs are now included in this category. However, a subset of brainstem gliomas lack K27M mutations and thus cannot be classified as any established glioma subtype. More data are needed to understand the clinical picture and underlying biology of these tumors.

In this study, biopsy and autopsy samples from previously diagnosed DIPG tumors were analyzed using next-generation sequencing (NGS) and immunohistochemistry (IHC). Our aims were as follows: (i) 
reclassify these tumors according to the WHO 2016 brain tumor classification and explore the correlation between clinical DIPG diagnosis and molecular diagnosis; (ii) screen the tumors for both known and as yet unstudied genetic alterations as well as pathological protein expression; (iii) explore tumor specimens of long-term survivors to find specific factors that can explain the unanticipated disease course; and (iv) develop an NGS panel applicable for diagnostic, therapeutic, and prognostic purposes for various pediatric brain neoplasms.

\section{2 | METHODS}

\section{1 | Patients and samples}

Diagnoses were made and the patients recruited to the study before publication of the WHO 2016 brain tumor classification. Thus, eligibility criteria are consistent with the clinical DIPG diagnosis and the WHO 2007 classification and include the following: (i) age younger than 16 years at diagnosis; (ii) radiologically verified DIPG (T1hypointense and T2-hyperintense tumor involving $\geq 50 \%$ of the pons) diagnosed at Children's Hospital, Helsinki University Central Hospital (HUCH), during 1994-2014; (iii) available tumor sample, obtained at diagnosis and/or autopsy, of sufficient amount and adequate quality for NGS and IHC; and (iv) a histological diagnosis of diffuse astrocytoma WHO grades II and III or glioblastoma grade IV. Patient demographics were collected retrospectively from hospital records with follow-up ending on October 25, 2015. The date of the first MRI was regarded as the day of diagnosis. The Institutional Review Board and the Ethics Committee approved the study design, and informed consent was received from patients still alive at the end of the follow-up.

\section{2 | Next-generation sequencing}

Tumor samples were analyzed with NGS utilizing a custom-made AmpliSeq panel, designed using AmpliSeq Designer pipeline Version 4.4.2 (Table 1, Supplementary Tables S1 and S2). For each sample, target regions were amplified in three multiplex polymerase chain reactions (PCRs). Three PCR pools were combined and prepared for libraries using Ion Xpress $^{\mathrm{TM}}$ Barcode Adapters and the lon AmpliSeq $^{\mathrm{TM}}$ Library Kit 2.0. Libraries were quantified using an Ion Library TaqMan ${ }^{\mathrm{TM}}$ Quantitation Kit and pooled in equimolar concentrations. Clonal amplification of the library pool was performed by emulsion PCR with lon PI ${ }^{\mathrm{TM}} \mathrm{Hi}-\mathrm{Q}^{\mathrm{TM}}$ OT2 $200 \mathrm{Kit}$ and Ion OneTouch ${ }^{\mathrm{TM}}$ 2 System. Templated ion sphere particles were enriched and then sequenced on Ion $\mathrm{PI}^{\mathrm{TM}}$ V3 Chips with an Ion $\mathrm{PI}^{\mathrm{TM}} \mathrm{Hi}-\mathrm{Q}^{\mathrm{TM}}$ Sequencing 200 Kit and an Ion Proton ${ }^{\mathrm{TM}}$ Sequencer. Sequence data were analyzed using Torrent Suite Software v4.6 and annotated via Ion Reporter software v4.6. NGS analysis was successful in 20 samples.

\section{3 | Immunohistochemistry}

IHC was utilized to analyze the protein expression of H3 K27M, TP53, IDH1 (R132H), SMARCB1 (INI1), CMYC, CTNNB1 (beta-catenin), EGFR, and CD31 (Supplementary File S1). IHC was unfeasible in two cases
TAB LE 1 Next-generation sequencing platform utilized in the study

\begin{tabular}{|c|c|c|c|}
\hline Gene & $\begin{array}{l}\text { Mutations (whole } \\
\text { codons) }\end{array}$ & $\begin{array}{l}\text { Mutations } \\
\text { (hotspots) }\end{array}$ & Amplifications \\
\hline ACVR1 & $x$ & & $x$ \\
\hline EGFR & $x$ & & $x$ \\
\hline $\mathrm{EZH} 2$ & $x$ & & $x$ \\
\hline KDR (VEGFR) & $x$ & & $x$ \\
\hline MET & $x$ & & $x$ \\
\hline PDGFRA & $x$ & & $x$ \\
\hline PPARG (PPAR $\gamma)$ & $x$ & & $x$ \\
\hline PTCH1 (PTCH) & $x$ & & $x$ \\
\hline SMARCA4 & $x$ & & $x$ \\
\hline$A L K$ & & $x$ & \\
\hline BRAF & & $x$ & \\
\hline CDKN2A & & $x$ & \\
\hline CTNNB1 & & $x$ & \\
\hline H3F3A & & $x$ & \\
\hline HIST1H3B & & $x$ & \\
\hline IDH1 & & $x$ & \\
\hline IDH2 & & $x$ & \\
\hline KRAS & & $x$ & \\
\hline PIКЗСА (PIЗКСА) & & $x$ & \\
\hline PTEN & & $x$ & \\
\hline SMARCB1 & & $x$ & \\
\hline SMO & & $x$ & \\
\hline TP53 & & $x$ & \\
\hline MYC (cMYC) & & & $x$ \\
\hline MYCN & & & $x$ \\
\hline
\end{tabular}

and the expression of K27M could not be analyzed in one additional biopsy due to technical issues. Consequently, IHC was conducted successfully in 24 samples for all targets except for H3 K27M, which was fulfilled in 23 samples.

Blinded for clinical data, an experienced neuropathologist evaluated the tumor histology and IHC staining results based on the proportion of immunopositive cells. H3 K27M, p53, IDH1, INI1, and nuclear beta-catenin expressions were classified as either positive or negative. For $\mathrm{p} 53$, nuclear expression in $>10 \%$ of tumor cells were considered positive and predictive for TP53 mutation. ${ }^{9}$ cMYC and EGFR expressions were graded on a four-step scale $(0=0 \%$; $1=1-25 \%$; $2=26$ $50 \%$; $3=>50 \%$ ). Further, the staining intensity of H3 K27M was evaluated as either high or low.

\section{4 | Microvessel density}

Several patients had received low-dose, continuous (metronomic) maintenance therapy according to the Angiocomb protocol, ${ }^{10}$ a treatment with antiangiogenic properties. Therefore, we also evaluated microvessel density (MVD) of the tumor samples as a separate part of the study. MVD was assessed utilizing the method developed by Weidner and based on the expression of CD3111 (Supplementary File S2). One biopsy showed DIPG infiltration of the cerebellum, and it was 
TAB LE 2 Patient characteristics

\begin{tabular}{|c|c|c|c|c|c|c|c|c|c|c|c|}
\hline \multirow[b]{2}{*}{ Patient } & \multirow[b]{2}{*}{ Age (yrs) } & \multicolumn{2}{|l|}{ Grade } & \multicolumn{2}{|c|}{ Therapy } & \multicolumn{2}{|c|}{ Outcome } & \multicolumn{2}{|c|}{ Biopsy } & \multicolumn{2}{|c|}{ Autopsy } \\
\hline & & Biopsy & Autopsy & RT & Other & Status & OS & NGS & $\mathrm{IHC}$ & NGS & $\mathrm{IHC}$ \\
\hline 1 & 8 & III & & Yes & $\begin{array}{l}\text { Vincristine, paclitaxel, } \\
\text { cyclophosphamide }\end{array}$ & DOD & 8.8 & $\mathrm{x}$ & $x$ & & \\
\hline 2 & 10 & III & & Yes & HSCT and autologous SCT & DOD & 15.4 & $x$ & $x$ & & \\
\hline 3 & 5 & IV & & Yes & $\begin{array}{l}\text { Vincristine, cisplatin, lomustine, } \\
\text { cyclophosphamide, paclitaxel }\end{array}$ & DOD & 10.3 & $x$ & $x$ & & \\
\hline 4 & 7 & III & & Yes & No & DOD & 12.0 & $x$ & $x$ & & \\
\hline 5 & 13 & II & & Yes & Angiocomb $b^{a}$ & AWD & 90.1 & $x$ & $x$ & & \\
\hline 6 & 15 & III & & Yes & No & DOD & 21.7 & $x$ & $x$ & & \\
\hline 7 & 5 & III & & Yes & No & DOD & 12.5 & & $\mathrm{x}$ & & \\
\hline 8 & 8 & IV & & Yes & Topotecan concurrently with RT & DOD & 5.4 & $x$ & $x$ & & \\
\hline 9 & 4 & II & & Yes & Angiocomb & $\mathrm{CR}$ & 47.8 & $\mathrm{x}$ & $x$ & & \\
\hline 10 & 15 & III & & Yes & Angiocomb & DOD & 7.4 & & $x$ & & \\
\hline 11 & 11 & III & & Yes & No & DOD & 9.3 & $x$ & $x$ & & \\
\hline 12 & 4 & II & & Yes & No & DOD & 13.6 & $x$ & $x$ & & \\
\hline 13 & 10 & III & & Yes & Angiocomb & DOD & 11.1 & $x$ & $x$ & & \\
\hline 14 & 4 & IV & & Yes & No & DOD & 3.8 & $x$ & & & \\
\hline 15 & 7 & II & & Yes & No & DOD & 15.1 & $x$ & & & \\
\hline 16 & 9 & IV & IV & Yes & Angiocomb & DOD & 7.2 & & $x$ & $x$ & $x$ \\
\hline 17 & 5 & III & IV & Yes & Angiocomb & DOD & 13.4 & & $x$ & $x$ & $x$ \\
\hline 18 & 11 & III & III-IV & Yes & Angiocomb $b^{b}$ & DOD & 5.2 & & $x$ & $x$ & $x$ \\
\hline 19 & 4 & & IV & No & 8-in-1 & DOD & 3.6 & & & $x$ & $x$ \\
\hline 20 & 7 & & IV & Yes & Angiocomb & DOD & 12.4 & & & $x$ & $x$ \\
\hline 21 & 2 & & IV & Yes & Angiocomb & DOD & 13.1 & & & $x$ & $x$ \\
\hline 22 & 12 & & IV & Yes & Angiocomb & DOD & 17.2 & & & $x$ & $x$ \\
\hline 23 & 10 & & IV & No & 8-in-1, HDCT and autologous SCT & DOD & 3.8 & & & & $x$ \\
\hline
\end{tabular}

Grade, histological grade according to WHO 2007 classification; yrs, years; RT, radiotherapy; OS, overall survival (months); NGS, next-generation sequencing; IHC, immunohistochemistry; DOD, died of disease; AWD, alive with disease; $C R$, complete response; SCT, stem cell transplantation; Angiocomb, radiotherapy and topotecan followed by metronomic thalidomide, etoposide, and celecoxib ${ }^{10} ; \mathrm{HDCT}$, high-dose chemotherapy; TMZ, temozolomide; BVA, bevacizumab; 8-in-1, vincristine, hydroxyurea, procarbazine, CCNU, cisplatin, cytarabine, high-dose methylprednisolone, cyclophosphamide.

${ }^{a}$ Only treatment given by pediatric neurooncologists.

${ }^{\text {b}}$ The patient had to discontinue thalidomide after approximately 1 week due to adverse events.

not assessable for MVD. Thus, MVD was evaluable in 23 samples. The MVDs of biopsy and autopsy samples were not comparable due to technical issues.

\section{5 | Statistical considerations}

The statistical analysis was conducted using IBM SPSS Statistics, Version 22 (SPSS, Chicago, IL, USA) and utilizing Kaplan-Meier survival analysis and Mann-Whitney $U$ test. Overall survival (OS) was defined as the period from diagnosis to death or the end of follow-up. In analyzing the relationship between MVD and survival, patients were dichotomized into two groups according to the median MVD. $P$-values $<0.05$ were considered significant.

\section{3 | RESULTS}

\section{1 | Patient demographics}

This study comprised 26 tumor samples, including 18 biopsies and 8 autopsy specimens, obtained from 23 patients (Table 2 and
Supplementary Table S3). Three patients had both biopsies and autopsy samples available. Of the 18 biopsies, 8 were stereotactic and 10 were obtained during a craniotomy and a microneurosurgical open biopsy because of the surgeon's preference. Altogether, three patients had postoperative complications (17\%), and all of these occurred after open biopsies. One patient had a mild but permanent facial nerve palsy and diplopia. One patient presented with transient ataxia, and one patient had transient worsening of a previous hemiparesis.

The median age at diagnosis was 8.3 years (range $2.7-15.3$ years), and 10 patients were females (44\%). At the end of follow-up, 21 of 23 patients (91\%) had died. The median OS was 12.0 months (range 3.690.1 months), and 12 and 24 months' survival rates were $52.2 \%$ and 8.7\%, respectively (Supplementary Table S4). Two patients were alive at the end of follow-up, 4.0 years and 7.5 years after diagnosis, respectively (Figs. 1A and 1B).

\section{2 | Alterations in H3 K27}

The most frequently discovered mutation in our study was H3 K27M, found in 20 of 23 patients (87\%) when including both NGS and IHC 

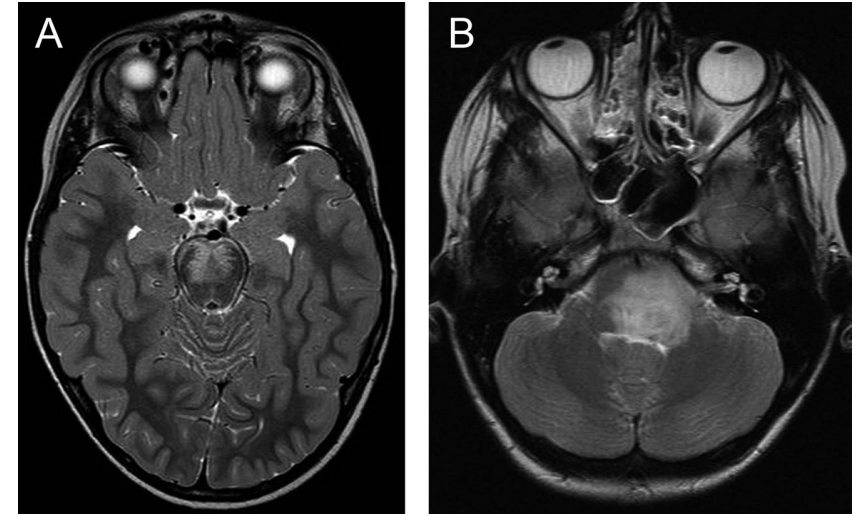

FIGURE 1 MRI from long-term survivors: (A) patient 9, OS 4.0 years and (B) patient 5 , OS 7.5 years at the end of follow-up

results (Table 3). Samples of 20 patients were analyzed by NGS and the $\mathrm{H} 3.3 \mathrm{~K} 27 \mathrm{M}$ mutation was found in 18 patients (90\%), whereas no $\mathrm{H} 3.1$ K27M mutation was encountered. IHC revealed H3 K27M expression in 18 of 21 patients (86\%) (Fig. 2A) with high staining intensity in 13 patients (72\%) and low intensity in five patients (28\%). Four of five samples with low K27M staining intensity were autopsy specimens. We did not find a clear correlation between $\mathrm{H} 3 \mathrm{~K} 27$ status and survival in our small cohort.

\section{3 | Alterations in TP53}

TP53 was the second most commonly altered gene in our cohort and was found in 10 patients (50\%) by NGS. Using IHC, 14 of 21 patients (67\%) expressed p53 in $>10 \%$ of the tumor cells. One of our patients did not express $\mathrm{p} 53$ by IHC although NGS analysis discovered a TP53 mutation (Fig. 2B), whereas four samples showed strong IHC positivity for p53, but lacked TP53 mutations in the NGS analysis (Fig. 2C).

\subsection{Other molecular alterations}

EGFR was mutated in one patient (5\%) by NGS. This patient lacked protein expression by IHC. Using IHC, EGFR expression was evident in six patients (29\%). None of these patients had EGFR mutations in NGS.

Three patients (15\%) had MET mutations, two patients (10\%) had mutations in PDGFRA, and one patient (5\%) a PDGFRA amplification. Additionally, two patients (10\%) had mutations in SMARCA4, one patient (5\%) had a mutation in PPAR $\gamma$, and one patient (5\%) a mutation in PTEN. VEGFR was mutated and amplified in one patient (5\%) each.

One of the long-term survivors with an OS of 7.5 years at the end of follow-up harbored an IDH1 mutation (R132S) in the diagnostic biopsy. Only NGS discovered the mutation, since the antibody utilized in IHC only recognizes the most common IDH1 alteration, which is $\mathrm{R} 132 \mathrm{H}$.

In addition, IHC revealed CMYC expression in 15 of 21 (71\%) patients (Fig. 2D). All positive specimens were biopsies. None of the autopsy samples expressed cMYC by IHC. We had three matched, biopsy-autopsy pairs from the same patients, and all biopsies were positive for $\mathrm{CMYC}$, while the autopsy pairs were negative. We did not find mutations or amplifications in CMYC by NGS.

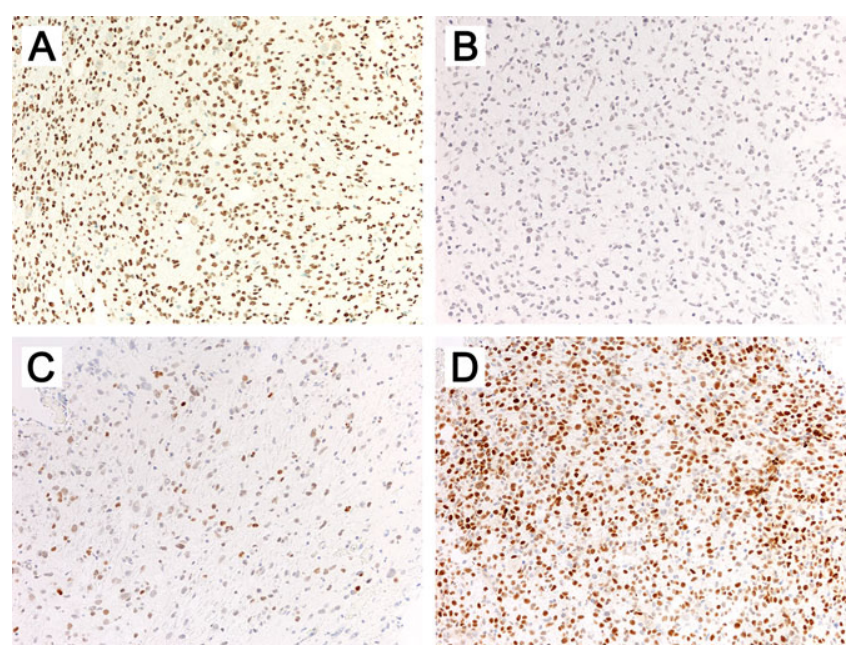

FIGURE 2 Immunohistochemical stainings (200× magnification), scale bar $100 \mu \mathrm{m}$ : (A) positive H3-K27M, (B) negative p53 despite of evident TP53 mutation by NGS, (C) positive p53 staining despite lack of TP53 mutation by NGS; (D) CMYC with strong expression ( $>50 \%$ of the cells stained)

\subsection{Microvessel density}

MVD showed a wide variation in both biopsies and autopsy samples. For biopsies, the difference between samples with the highest and lowest MVD was a six-folded (range $30-179 / \mathrm{mm}^{2}$, median $64 / \mathrm{mm}^{2}$ ), and for autopsy specimens, a three-folded (range 60-186/ $\mathrm{mm}^{2}$, median $120 / \mathrm{mm}^{2}$ ) (Supplementary Table S5). None of these alterations correlated with survival.

\section{4 | DISCUSSION}

DIPG is a well-known clinical diagnosis. In the WHO 2007 brain tumor classification, ${ }^{12}$ DIPGs were categorized based on their histology as diffuse astrocytomas. Recent molecular studies have revealed that a significant share of DIPGs bear a K27M mutation in histone 3. 3,4,6,7,13-16 Together with other diffuse midline H3 K27M-mutated gliomas, these tumors now constitute a new entity in the revised $\mathrm{WHO}$ 2016 brain tumor classification known as diffuse midline glioma, H3 K27M-mutant. ${ }^{8}$ These tumors are associated with the most aggressive disease and the most dismal prognosis. ${ }^{2}$

In earlier DIPG studies, the K27M mutation has resided in histone 3.3 in $48-71 \%$ of the cases, ${ }^{2-7,17}$ whereas H3.1-K27M mutations occur at a frequency of $11-25 \% .^{2-5,17}$ In our cohort, $87 \%$ of the patients diagnosed with a DIPG based on clinical findings also harbored a H3 K27 mutation. Thus, in our cohort, brainstem tumors with symptoms and a radiological presentation characteristic for DIPG most often belonged to a molecular entity of $\mathrm{H} 3 \mathrm{~K} 27$-mutated diffuse midline gliomas, showing that careful clinical DIPG diagnosis usually correlates with a molecular diagnosis of aggressive H3 K27M-mutated gliomas.

Our study cohort included three patients (13\%) with wild-type H3 K27. The OS in this group did not statistically differ significantly from 
TAB LE 3 Results of exon sequencing and immunohistochemistry

\begin{tabular}{|c|c|c|c|c|c|c|c|c|c|}
\hline \multirow[b]{2}{*}{ ID } & \multirow[b]{2}{*}{ OS (months) } & \multirow[b]{2}{*}{ Sample } & \multicolumn{2}{|c|}{ K27M } & \multicolumn{2}{|l|}{ TP53 } & \multirow{2}{*}{$\begin{array}{l}\text { cMYC } \\
\text { IHC }\end{array}$} & \multirow{2}{*}{$\begin{array}{l}\text { EGFR } \\
\text { IHC }\end{array}$} & \multirow[b]{2}{*}{ Other mutations } \\
\hline & & & NGS & IHC & NGS & $\mathrm{IHC}$ & & & \\
\hline 1 & 8.8 & Biopsy & H3.3 & + & Mutation & + & 1 & 0 & 0 \\
\hline 2 & 15.4 & Biopsy & H3.3 & + & Mutation & - & 2 & 0 & PDGFRA \\
\hline 3 & 10.3 & Biopsy & H3.3 & + & WT & - & 0 & 0 & ACVR1, MET \\
\hline 4 & 12.0 & Biopsy & H3.3 & + & Mutation & + & 1 & 0 & SMARCA4 \\
\hline 5 & 90.1 & Biopsy & WT & - & Mutation & + & 1 & 0 & $I D H 1^{a}$ \\
\hline 6 & 21.7 & Biopsy & H3.3 & + & WT & + & 1 & 0 & 0 \\
\hline 7 & 12.5 & Biopsy & NA & + & NA & + & 3 & 0 & NA \\
\hline 8 & 5.4 & Biopsy & H3.3 & + & Mutation & + & 3 & 0 & 0 \\
\hline $9^{2}$ & 47.8 & Biopsy & WT & - & WT & - & 1 & 0 & 0 \\
\hline 10 & 7.4 & Biopsy & NA & + & NA & + & 3 & 1 & NA \\
\hline 11 & 9.3 & Biopsy & H3.3 & + & WT & - & 1 & 2 & 0 \\
\hline 12 & 13.6 & Biopsy & H3.3 & + & WT & + & 3 & 0 & 0 \\
\hline 13 & 11.1 & Biopsy & H3.3 & + & Mutation & + & 1 & 0 & PTEN \\
\hline 14 & 3.8 & Biopsy & H3.3 & NA & WT & NA & NA & NA & 0 \\
\hline 15 & 15.1 & Biopsy & H3.3 & NA & Mutation & NA & NA & NA & MET \\
\hline \multirow[t]{2}{*}{16} & 7.2 & Biopsy & NA & NA & NA & + & 2 & 0 & NA \\
\hline & & Autopsy & H3.3 & + & Mutation & + & 0 & 1 & 0 \\
\hline \multirow[t]{2}{*}{17} & 13.4 & Biopsy & NA & + & NA & - & 3 & 1 & NA \\
\hline & & Autopsy & H3.3 & + & WT & - & 0 & 1 & 0 \\
\hline \multirow[t]{2}{*}{18} & 5.2 & Biopsy & NA & + & NA & + & 1 & 0 & NA \\
\hline & & Autopsy & H3.3 & + & WT & + & 0 & 1 & PDGFRA, PPAR $\gamma^{3}, \mathrm{MET}^{3}$ \\
\hline 19 & 3.6 & Autopsy & H3.3 & + & Mutation & + & 0 & 0 & 0 \\
\hline 20 & 12.4 & Autopsy & H3.3 & + & Mutation & + & 0 & 1 & 0 \\
\hline 21 & 13.1 & Autopsy & H3.3 & + & WT & + & 0 & 0 & EGFR \\
\hline 22 & 17.2 & Autopsy & H3.3 & + & WT & - & 0 & 0 & VEGFR, SMARCA4 \\
\hline 23 & 3.8 & Autopsy & NA & - & NA & - & 0 & 0 & NA \\
\hline
\end{tabular}

All tumors were clinically and radiologically diagnosed as DIPGs at baseline. Patient 14 had also amplifications of PDGFRA and VEGFR (not included in the table). ID, patient identification number; OS, overall survival; NGS, next-generation sequencing; IHC, immunohistochemistry; WT, wild type; NA, not assessable.

a Immunohistochemistry of IDH1 was negative.

${ }^{2}$ The proportion of tumor cells was $<10 \%$, which may influence the sequencing results.

${ }^{3}$ Strong indication of mutations.

those patients who were positive for H3 K27M. However, this finding may be due to the small cohort size, which prevents a reliable analysis. Interestingly, one of the patients lacking the H3 K27 mutation experienced a rapid disease course and survived for only 3.8 months. This finding was unexpected, as tumors harboring $\mathrm{H} 3 \mathrm{~K} 27 \mathrm{M}$, and not the wild-type $\mathrm{H} 3 \mathrm{~K} 27$, have been associated with the worst prognosis. ${ }^{2}$ The reason for this fast tumor progression is equivocal. The fact that no radiation therapy was given to this patient may also have influenced the disease course. Nonetheless, there may be other, yet unknown, genetic factors that affect the outcomes of patients without H3 K27 mutations.

The other patients who were negative for $\mathrm{H} 3 \mathrm{~K} 27 \mathrm{M}$ mutations were two long-term survivors who were alive at the end of follow-up, 4.0 and 7.5 years from diagnosis, respectively. Both were initially diagnosed to have DIPG at their baseline according to radiological and histolog- ical findings. However, current analyses discovered features that warranted a reevaluation of these diagnoses.

In the first patient, the initial radiological diagnosis was DIPG. The patient was included in this study according to the initial diagnosis. However, when reevaluated for this study, the MRI images of this patient showed distinctive features compared to other DIPGs (Supplementary Figs. S1-S3): the lesion has diffuse boundary to the normal tissue, but it is smaller and the mass effect is slighter than in other patients. Histology of the biopsied lesion was consistent with grade II astrocytoma infiltration zone. Unfortunately, NGS was impossible to conduct due to the low tumor cell content of the tumor sample $(<10 \%)$, and we were thus not able to confirm the molecular diagnosis of this particular tumor. This patient received treatment according to the antiangiogenic Angiocomb protocol. ${ }^{10}$ After radiotherapy with concomitant topotecan infusions, the tumor disappeared radiologically, and follow-up MRIs confirmed a sustained complete response. ${ }^{10}$ 
When taking into account the radiological reassessment and the lack of molecular findings, it now seems unlikely that this patient had a real DIPG. Consequently, combined radiological, histological, and molecular diagnosis is essential to confirm the clinical diagnosis and would have been of highest importance in this case. This is the main message of our study.

The other long-term survivor had a typical MRI appearance of the tumor at diagnosis (Supplementary Figs. S4-S9). However, current molecular findings in the biopsy indicate that this tumor was not a classic aggressive DIPG. An IDH1 mutation was discovered by NGS in the biopsy. IHC was unable to detect this alteration, as the antibody only recognizes the most common mutation, $\mathrm{R} 132 \mathrm{H} .{ }^{18}$ The R132S mutation found in our patient has prevalence of only $1.4 \%$ in all IDH1/2-mutated gliomas. ${ }^{18}$ In the revised WHO 2016 tumor classification, the IDH mutation status divides diffuse gliomas into further subgroups. ${ }^{8}$ Mutations in IDH1 or IDH2 occur in approximately $10 \%$ of glioblastomas, ${ }^{8}$ predominately among adolescents and in cortical tumors. ${ }^{19}$ IDH1 mutations confer a favorable prognostic marker that is associated with extended survival in supratentorial gliomas, ${ }^{18,20}$ but these have thus far been regarded as being absent in pediatric brainstem astrocytomas. 2,4,6,7,21,22 The IDH1 mutation found in our second long-term survivor now warrants a reevaluation of this paradigm. Furthermore, this finding also indicates that a molecular diagnosis of a biopsy is needed to confirm the real nature of a brainstem tumor despite its typical clinical and radiological presentation.

By NGS, TP53 mutations were found in 10 patients (50\%), a prevalence similar to those reported in most earlier studies on DIPGs. $6,7,23$ There was some discrepancy between the NGS and the IHC results. One patient was negative for $\mathrm{p} 53$ by IHC, although NGS did discover a TP53 mutation. This result is not unforeseen, since NGS is superior to IHC in both sensitivity and specificity, and truncating TP53 mutations are not recognized by IHC. ${ }^{9}$ Four samples showed strong IHC positivity for p53, but lacked TP53 mutations in an NGS analysis. Pollack et al. ${ }^{24}$ proposed that TP53 overexpression without gene mutation can occur as a stress-related response to anoxia and DNA damage. Moreover, we cannot exclude the fact that the old age of some samples may have yielded false-negative TP53 mutation status. The rather high degree of inconsistency in the NGS and IHC results was evident despite the utilization of a $10 \%$ cutoff for positive and negative $\mathrm{IHC}$ results, a criterion that is considered superior to previous classifications. ${ }^{9}$ Thus, although IHC is the standard method for screening TP53 mutations in many laboratories, a parallel genetic approach would be preferable.

The tyrosine kinase receptors PDGFRA, EGFR, and VEGFR as well as the $\mathrm{mTOR}$ pathway have been suggested as potential targets for novel treatments of brainstem gliomas. The presence of these targets in diagnostic biopsies would, therefore, be of great interest. In previous studies, PDGFRA amplification/gain or mutations have been encountered quite frequently in DIPG. 2,6,14,23,25-28 In our cohort, two patients had mutations in PDGFRA, and one patient a PDGFRA amplification. EGFR amplifications and mutations, common in adult glioblastomas, ${ }^{29}$ are rare in DIPGs. 2,26,28 Interestingly, we found an EGFR mutation in one of our DIPG patients who also had a H3 K27M mutation. This tumor lacked EGFR expression by IHC, which may reflect the incapability of the antibody to recognize the mutated form of EGFR. EGFR expression was found in six patients, but none of these harbored EGFR mutations or amplifications.

Consistent with our study, other studies have also reported a lack of association between EGFR gene alterations and protein expression. 2,28 Regarding the mTOR pathway, MET ${ }^{30}$ and PTEN ${ }^{7}$ mutations have been reported to occur only sporadically in DIPGs. Alterations in VEGFR are rare. However, in our cohort, VEGFR was mutated and amplified in one patient each. In all, our results are largely consistent with earlier studies, but the proportion of rare EGFR and VEGFR alterations were higher than previously reported.

Two patients harbored SMARCA4 mutations. SMARCA4 encodes a component of the chromatin remodeling complex SWI/SNF, and mutations in this gene distinguish a minority of atypical teratoid/rhabdoid tumors (AT/RTs). ${ }^{31}$ In addition, one patient had a mutation in PPAR a multifunctional nuclear receptor involved in insulin and glucose metabolism, inflammatory processes, and immune responses. ${ }^{32}$ There is preliminary evidence of its role in adult gliomagenesis, and PPAR $\gamma$ protein expression levels have been reported to correlate inversely with the histological grade. ${ }^{32}$ Further, PPAR $\gamma$ agonists have demonstrated antitumor and antiangiogenic effects in preclinical studies. ${ }^{32}$ The functional impact of SMARCA4 and PPAR $\gamma$ mutations in diffuse brainstem gliomas, however, remains unclear.

Amplification of the oncogene $C M Y C$ is associated with an inferior prognosis in other tumors, such as medulloblastomas. ${ }^{33}$ The correlation of CMYC amplification to prognosis in DIPG is yet not established. In our study, none of the tumors had $\mathrm{CMYC}$ amplification, but protein expression was evident in 15 patients (71\%), excluding the long-term survivors. It is known that cMYC can be overexpressed also without gene amplification, such as through translocations. ${ }^{34-36}$ Interestingly, cMYC overexpression was found exclusively in the biopsy samples. This finding also applies to the three matched biopsy-autopsy pairs in this study; biopsies were positive for cMYC expression while autopsy samples from the same patients were negative. Technical issues related to tissue preservation at autopsy may have interfered with the results, but the high degree of $C M Y C$ positivity in the biopsies and the lack of expression in the autopsy samples may also indicate that some factor-potentially radiotherapy, which most of the patients received-could have eradicated the $C M Y C$-positive tumor cells while leaving treatment-resistant subclones remaining. cMYC expression did not correlate with survival in our cohort, but it would be interesting to validate the significance in a larger patient group.

Angiogenesis-related pathways have been proposed to be crucial in a subset of DIPGs. ${ }^{3,37}$ How differences in angiogenesis signaling are reflected on the histological level is still unknown, causing us to study the MVD in our tumor samples. Due to the scarce size of the biopsies, we had to utilize different methods to evaluate the MVD in the biopsies and autopsy samples. The MVD showed a wide variation in both biopsies and autopsy samples, but it did not correlate with survival or with the utilization of metronomic therapy.

There are certain caveats associated with our study. Substantial tumor heterogeneity for certain genetic alterations ${ }^{26,30}$ increases the risk of sampling bias. Due to the small number of samples, we were unable to conduct more genetic analyses or find any statistically significant correlations to survival. 
In conclusion, molecular analysis of brainstem gliomas previously diagnosed as DIPGs revealed H3 K27M mutations in most of the cases. DIPGs with a typical MRI appearance most often corresponded to diffuse midline glioma, H3 K27-mutant. However, three patients (13\%) did not fit into this category. One of these patients was a long-term survivor who harbored an IDH1 mutation, a novel finding in pediatric brainstem gliomas, which implies that this tumor was not a classic DIPG. Thus, clinical and radiological diagnosis is not always sufficient to categorize brainstem tumors. Nowadays more and more subjects undergo a biopsy, and the combined histological and molecular findings should be taken into consideration when making the final diagnosis.

\section{ACKNOWLEDGMENTS}

We thank Leena Saikko, Mia Kero, Soili Kytölä, Arto Orpana, AnnaKaisa Anttonen, Hanna Tammio, Johanna Yli-Sikkilä, and Milja Kaare for their excellent technical support; Anders Paetau and Maarit Palomäki for expert diagnostic help; and the $\mathrm{HUCH}$ Department of Neurosurgery for the biopsies.

\section{CONFLICT OF INTEREST}

The authors declare that there is no conflict of interest.

\section{REFERENCES}

1. Warren KE. Diffuse intrinsic pontine glioma: poised for progress. Front. Oncol. 2012;2:1-9.

2. Buczkowicz P, Hawkins C. Pathology, molecular genetics, and epigenetics of diffuse intrinsic pontine glioma. Front. Oncol. 2015;5:1-9.

3. Castel D, Philippe C, Calmon R, et al. Histone H3F3A and HIST1H3B K27M mutations define two subgroups of diffuse intrinsic pontine gliomas with different prognosis and phenotypes. Acta Neuropathol. 2015;130:815-827.

4. Khuong-Quang DA, Buczkowicz P, Rakopoulos $P$, et al. K27M mutation in histone $\mathrm{H} 3.3$ defines clinically and biologically distinct subgroups of pediatric diffuse intrinsic pontine gliomas. Acta Neuropathol. 2012;124:439-447.

5. Wu G, Broniscer A, McEachron TA, et al. Somatic histone H3 alterations in pediatric diffuse intrinsic pontine gliomas and non-brainstem glioblastomas. Nat. Genet. 2012;44:251-253.

6. Buczkowicz P, Hoeman C, Rakopoulos P, et al. Genomic analysis of diffuse intrinsic pontine gliomas identifies three molecular subgroups and recurrent activating ACVR1 mutations. Nat. Genet. 2014;46:451-456.

7. Taylor KR, Mackay A, Truffaux N, et al. Recurrent activating ACVR1 mutations in diffuse intrinsic pontine glioma. Nat. Genet. 2014;46:457461.

8. Louis DN, Ohgaki H, Wiestler OD, Cavenee WK. WHO Classification of Tumors in the Central Nervous System. Revised 4th ed. Lyon: IARC 2016.

9. Takami H, Yoshida A, Fukushima S, et al. Revisiting TP53 mutations and immunohistochemistry-a comparative study in 157 diffuse gliomas. Brain Pathol. 2015;25:256-265.

10. Porkholm M, Valanne L, Lonnqvist T, et al. Radiation therapy and concurrent topotecan followed by maintenance triple anti-angiogenic therapy with thalidomide, etoposide, and celecoxib for pediatric diffuse intrinsic pontine glioma. Pediatr. Blood Cancer. 2014;61:16031609.
11. Weidner N. Measuring intratumoral microvessel density. Methods Enzymol. 2008;444:305-323.

12. Louis DN, Ohgaki H, Wiestler OD, Cavenee WK, eds. WHO Classification of Tumours of the Central Nervous System. Lyon: IAARC; 2007.

13. Schwartzentruber J, Korshunov A, Liu XY, et al. Driver mutations in histone $\mathrm{H} 3.3$ and chromatin remodelling genes in paediatric glioblastoma. Nature. 2012;482:226-231.

14. Wu G, Diaz AK, Paugh BS, et al. The genomic landscape of diffuse intrinsic pontine glioma and pediatric non-brainstem high-grade glioma. Nat. Genet. 2014;46:444-450.

15. Fontebasso AM, Papillon-Cavanagh S, Schwartzentruber J, et al. Recurrent somatic mutations in ACVR1 in pediatric midline high-grade astrocytoma. Nat. Genet. 2014;46:462-466.

16. Grasso CS, Tang Y, Truffaux N, et al. Functionally defined therapeutic targets in diffuse intrinsic pontine glioma. Nat. Med. 2015;21:555559.

17. Buczkowicz P, Bartels U, Bouffet E, Becher O, Hawkins C. Histopathological spectrum of paediatric diffuse intrinsic pontine glioma: diagnostic and therapeutic implications. Acta Neuropathol. 2014;128:573581.

18. Kato Y. Specific monoclonal antibodies against IDH1/2 mutations as diagnostic tools for gliomas. Brain Tumor Pathol. 2015;32:3-11.

19. Fontebasso AM, Liu XY, Sturm D, Jabado N. Chromatin remodeling defects in pediatric and young adult glioblastoma: a tale of a variant histone 3 tail. Brain Pathol. 2013;23:210-216.

20. Sturm D, Witt $H$, Hovestadt $V$, et al. Hotspot mutations in H3F3A and IDH1 define distinct epigenetic and biological subgroups of glioblastoma. Cancer Cell. 2012;22:425-437.

21. Solomon DA, Wood MD, Tihan T, et al. Diffuse midline gliomas with histone H3-K27M mutation: a series of 47 cases assessing the spectrum of morphologic variation and associated genetic alterations. Brain Pathol. 2016;26:569-580.

22. Ballester LY, Wang Z, Shandilya S, et al. Morphologic characteristics and immunohistochemical profile of diffuse intrinsic pontine gliomas. Am J Surg Pathol. 2013;37:1357-1364.

23. Paugh BS, Zhu X, Qu C, et al. Novel oncogenic PDGFRA mutations in pediatric high-grade gliomas. Cancer Res. 2013;73:6219-6229.

24. Pollack IF, Hamilton RL, Finkelstein SD, et al. The relationship between TP53 mutations and overexpression of $\mathrm{p} 53$ and prognosis in malignant gliomas of childhood. Cancer Res. 1997;57:304-309.

25. Barrow J, Adamowicz-Brice M, Cartmill M, et al. Homozygous loss of ADAM3A revealed by genome-wide analysis of pediatric highgrade glioma and diffuse intrinsic pontine gliomas. Neuro Oncol. 2011;13:212-222.

26. Paugh BS, Broniscer A, Qu C, et al. Genome-wide analyses identify recurrent amplifications of receptor tyrosine kinases and cellcycle regulatory genes in diffuse intrinsic pontine glioma. $J$ Clin Oncol. 2011:29:3999-4006.

27. Paugh BS, Qu C, Jones C, et al. Integrated molecular genetic profiling of pediatric high-grade gliomas reveals key differences with the adult disease. J Clin Oncol. 2010;28:3061-3068.

28. Zarghooni M, Bartels U, Lee E, et al. Whole-genome profiling of pediatric diffuse intrinsic pontine gliomas highlights platelet-derived growth factor receptor alpha and poly (ADP-ribose) polymerase as potential therapeutic targets. J Clin Oncol. 2010;28:1337-1344.

29. Huse JT, Aldape KD. The evolving role of molecular markers in the diagnosis and management of diffuse glioma. Clin Cancer Res. 2014:20:5601-5611.

30. Hoffman LM, DeWire M, Ryall S, et al. Spatial genomic heterogeneity in diffuse intrinsic pontine and midline high-grade glioma: implications 
for diagnostic biopsy and targeted therapeutics. Acta Neuropathol Commun. 2016;4:1-8.

31. Fruhwald MC, Biegel JA, Bourdeaut F, Roberts CW, Chi SN. Atypical teratoid/rhabdoid tumors-current concepts, advances in biology, and potential future therapies. Neuro Oncol. 2016;18:764-778.

32. Zhang J, Yang W, Zhao D, et al. Correlation between TSP-1, TGF-beta and PPAR-gamma expression levels and glioma microvascular density. Oncol Lett. 2014;7:95-100.

33. Massimino M, Biassoni V, Gandola L, et al. Childhood medulloblastoma. Crit Rev Oncol Hematol. 2016;105:35-51.

34. Stine ZE, Walton ZE, Altman BJ, Hsieh AL, Dang CV. MYC, metabolism, and cancer. Cancer Discov. 2015;5:1024-1039.

35. Dang C. MYC on the path to cancer. Cell. 2012;149:22-35.

36. Wahlström T, Arsenian Henriksson M. Impact of MYC in regulation of tumor cell metabolism. Biochim Biophys Acta. 2015;1849:563-569.
37. Puget S, Philippe C, Bax DA, et al. Mesenchymal transition and PDGFRA amplification/mutation are key distinct oncogenic events in pediatric diffuse intrinsic pontine gliomas. PLoS One. 2012;7:e30313.

\section{SUPPORTING INFORMATION}

Additional Supporting Information may be found online in the supporting information tab for this article.

How to cite this article: Porkholm M, Raunio A, Vainionpää R, et al. Molecular alterations in pediatric brainstem gliomas. Pediatr Blood Cancer. 2018;65:e26751. https://doi.org/10.1002/ pbc.26751 